\title{
Pedagogía de la Educación Física: análisis de las revistas españolas más productivas (2005-2014) Physical Education Pedagogy: analysis of the most productive Spanish journals (2005-2014) Pere Molina,Alejandro Martínez-Baena, Miguel Villamón Universidad de Valencia (España)
}

Resumen. El propósito de este estudio es conocer el estado de la investigación sobre Pedagogía de la Educación Física en España, a partir del análisis de las cuatro revistas nacionales más productivas de este campo en la última década (2005-2014). Las revistas han publicado un total de 929 artículos, 400 de ellos (un 43,06\%) eran estudios relacionados con la pedagogía de la Educación Física. De este grupo, un 47,75\% se ocupaban de la enseñanza, un $27,75 \%$ de cuestiones relacionadas con el currículum de esta materia escolar, un 9,5\% integraban ambas subáreas y un 8,75\% se referían a la formación del profesorado. En cuanto al tipo de artículos, un 46,25\% eran estudios teóricos, históricos o ensayos, un 28\% trataban de experiencias educativas o de innovación y un $25 \%$ se correspondía con investigaciones empíricas. Los resultados presentan una gran diferencia con respecto a los obtenidos en estudios similares realizados en países anglosajones. Se concluye advirtiendo la necesidad de incrementar los porcentajes de artículos originales de investigación empírica (cuantitativa, cualitativa o mixta).

Palabras clave. Scopus, Web of Science, tendencias de investigación, Ciencias del Deporte, Pedagogía, Educación Física

Abstract. The purpose of this study is to know the state of research in Physical Education Pedagogy in Spain, based on the analysis of the four most productive national journals in this field during the last decade (2005-2014). Journals published a total of 929 papers, 400 of them (43.06\%) are related to the Physical Education Pedagogy. From this group, 47.75\% was focused on teaching, 27.75\% on curriculum issues, 9.5\% combined both possibilities and $8.75 \%$ referred to teacher education. Regarding the type of article, $46.25 \%$ were theoretical, historical studies or essays, $28 \%$ dealt with educational experiences or innovation and $25 \%$ included empirical research. The results show a great difference with the Anglo-Saxon countries. The study concludes that an increase of empirical research articles of different kind was necessary (quantitative, qualitative or mixed).

Keywords. Scopus, Web of Science, research trends, Sports Science, Pedagogy, Physical Education.

\section{Introducción}

En el contexto académico español el término Educación Física se ha utilizado tradicionalmente en un sentido amplio que integraba el conjunto de prácticas físico-deportivas, vinculadas con una preocupación pedagógica. Ala asunción de esta forma de entender la Educación Física contribuyó la concepción e influencia de José María Cagigal y la creación de los primeros centros de educación superior bajo la denominación de Institutos Nacionales de Educación Física. En 1992, la integración de estos estudios en la Universidad (MEC, 1992), conllevó también que, al año siguiente, se cambiase la denominación de los estudios de Educación Física por la Licenciatura en Ciencias de la Actividad Física y del Deporte(CCAFD) (MEC, 1993). Se advierte en ello cuatro cambios académicos importantes: ampliar los límites del objeto de estudio a la actividad física y el deporte, aportar nuevas orientaciones en la práctica físico-deportiva (además de la pedagógica), subrayar el carácter científico de estos estudios y asumir una visión interdisciplinar y aplicada de la actividad física y el deporte para ser estudiada desde las diversas ciencias. La intención era, en definitiva, ampliar los ámbitos de actuación profesional y dotar de una formación de carácter científico a los titulados en CCAFD.

La integración de los estudios en la Universidad española y la orientación científica que toman exigen al profesorado del área de Educación Física y Deportiva una reorientación de su perfil académicopráctico como profesor de educación física/entrenador de deporte a un perfil académico-científico como investigador en actividad física y deporte. Se entiende que el profesorado necesita investigar para consolidar su puesto de trabajo y desarrollarse profesionalmente. Como primer requisito destaca la necesaria consecución del grado de Doctor. Ante la falta de programas específicos, muchos de estos profesores se integran, como estudiantes de tercer ciclo, en los programas de otras áreas (psicología y fisiología, principalmente). Este profesorado de educación física/ entrenador de deporte comienza así una nueva trayectoria académicocientífica relacionada con los aspectos psicológicos y biomédicos de la actividad física y el deporte. Se comienzan también a solicitar proyectos de investigación a organismos oficiales.

Paralelamente, a principios de la década de 1990, en los estudios de

Fecha recepción: 31-07-15. Fecha de aceptación: 07-05-16 Fecha recepcion:
Pere Molina

juan.p.molina@uv.es
Magisterio se crea la especialidad (ahora denominada mención) de Educación Física y con ella la aparición de materias que requieren de profesorado especialista del área de Expresión Corporal (MEC, 1991). Por su parte, los estudios en CCAFD viven un momento de expansión en la oferta universitaria, tanto pública como privada, pasando de 8 centros, a principios del 2000, a más de 30, a finales de dicha década. La investigación deja de ser una opción para convertirse en una exigencia para el desarrollo profesional del profesorado universitario. Vinculado a ello, se genera también la necesidad de revistas para publicar los, cada vez más abundantes, trabajos de investigación.

En la década de 1990 y primera mitad de la del 2000, surgen la mayor parte de las revistas de carácter científico del ámbito de la actividad física y deporte que se publican actualmente en España. Algunas optan por un carácter interdisciplinar y otras se especializan en ciencias aplicadas al estudio de la actividad física y el deporte, como la psicología y la medicina (Valenciano, Devís \& Beltrán, 2008).

A su vez, se crean en España las agencias evaluadoras de la actividad investigadora: la Comisión Nacional Evaluadora de la Actividad Investigadora (CENAI), en 1989, y la Agencia Nacional de Evaluación de la Calidad y la Acreditación (ANECA), en 2002, que utilizan como principales criterios, para la evaluación de la actividad investigadora del profesorado universitario, la publicación de artículos en revistas indizadas en la Web of Science ${ }^{\circledR}$ (WoS) y, más recientemente, Scopus ${ }^{\circledR}$ (Villamón, Job, Valenciano \& Devís (2012). Tan importante es para las revistas integrarse en bases de datos internacionales que, desde 2007, la Fundación Española para la Ciencia y la Tecnología (FECYT) realiza una convocatoria bienal para la concesión del sello de calidad para revistas españolas con la intención de potenciar su presencia en las bases de

Tabla 1.

Revistas españolas de CCAFD indizadas en WoS, Scopus o con sello de calidad de la FECYT en 2015

\begin{tabular}{ll}
\hline Revista: & Índice de calidad: \\
\hline Apunts. Educación Física y Deportes & Scopus, Sello FECYT \\
\hline Apunts. Medicina de l'Esport & Scopus, Sello FECYT \\
\hline Archivos de Medicina del Deporte & Scopus \\
\hline Cuadernos de Psicología del Deporte & WoS, Scopus \\
\hline Cultura, Ciencia y Deporte & Scopus, Sello FECYT \\
\hline Journal of Human Sport \& Exercise & Scopus \\
\hline $\begin{array}{l}\text { Retos. Nuevas Tendencias en Educación Física, Deporte y } \\
\text { Recreación }\end{array}$ & Sello FECYT \\
\hline Revista Andaluza de Medicina del Deporte & Scopus \\
\hline Revista de Psicología del Deporte & WoS, Scopus \\
\hline $\begin{array}{l}\text { Revista Iberoamericana de Psicología del Ejercicio y del } \\
\text { Deporte }\end{array}$ & Scopus \\
\hline RICYDE. Revista Internacional de Ciencias del Deporte & Scopus, Sello FECYT \\
\hline $\begin{array}{l}\text { Revista Internacional de Medicina y Ciencias de la Actividad } \\
\text { Física y el Deporte }\end{array}$ & WoS, Scopus, Sello FECYT \\
\hline
\end{tabular}


datos internacionales. Los artículos publicados en revistas acreditadas con este sello también se tienen en cuenta dentro de los criterios en la evaluación de la actividad investigadora. Por tanto, las aportaciones en revistas indizadas en WoS, Scopus o que reciban el sello de calidad de la FECYT son las que se valoran preferentemente dentro de la actividad investigadora del profesorado universitario. En la tabla 1 se recogen las revistas españolas que cumplen alguno de estos criterios.

La mitad de las revistas de la tabla 1 tiene un carácter interdisciplinar y la otra mitad son revistas disciplinares vinculadas con las áreas de la psicología o la medicina. Aunque en muchas de ellas tienen cabida artículos relacionados con el ámbito pedagógico, sólo Retos. Nuevas Tendencias en Educación Física, Deporte y Recreación, mantiene una vinculación directa con esta disciplina, pues está editada por la Federación Española de Asociaciones Docentes de Educación Física (FEADEF). La carencia de revistas científicas disciplinares pedagógicas en el campo de las CCAFD puede tener consecuencias importantes en el estatus científico de la Pedagogía de la Educación Física (Physical Education Pedagogy). Una de ellas puede ser que muchos investigadores, sobre todo noveles, se sientan más atraídos por los ámbitos de conocimiento con mayor presencia en las revistas indizadas en $W o S$ o Scopus, porque publicar en ellas tiene consecuencias positivas en Su currículum. Más aún si en la acreditación para algunas figuras de profesorado universitario, como ayudante doctor y contratado doctor, no se distinguen perfiles, como sucede actualmente. De todo esto, se puede deducir que los aspirantes a profesores y profesoras de algunos ámbitos de conocimiento, como el pedagógico, lo van a tener más complicado, de partida, para su desarrollo profesional investigador que otros ámbitos más consolidados y atendidos. Esto provocará a corto plazo (se está viviendo ya) una descompensación en los ámbitos de investigación en las CCAFD; unos con muchos especialistas y gran volumen de producción, y otros con pocos especialistas y menor productividad. Una consecuencia para los planes de estudio y la formación de graduados en CCAFD será la falta de profesorado especialista en determinados ámbitos y la impartición de materias por profesorado que tiene su docencia en ellas pero que, sin embargo, investiga en otros ámbitos donde encuentra una mayor proyección. En el caso de los Grados de Magisterio y el área de Expresión Corporal, la situación puede ser más aguda, si cabe.

Esta situación académico-científica del ámbito pedagógico requiere de un estudio de las revistas españolas más productivas en esta disciplina que permita conocer su realidad y servir de orientación, tanto para los investigadores en este ámbito como para los equipos editoriales de las mismas. En el panorama internacional encontramos diversos trabajos que han analizado la productividad en el ámbito de la Pedagogía de la Educación Física(PEF) de las principales revistas científicas (Hemphill., Richards, Templin \& Blankenship, 2012; Kirk, 2010; Kulinna, ScrabisFletcher, Kodish, Philips \& Silverman, 2009; Silverman \& Skonie, 1997; Ward \& Ko, 2006). Entre ellos cabe destacar los realizados por los editores de dos de las más relevantes en este ámbito: uno encabezado por Pamela H. Kulinna, editora entre 2010 y 2014 de Journal of Teaching in Physical Education y otro de David Kirk, actual editor de Physical Education and Sport Pedagogy.

Kulinna et al. (2009) realizan una revisión general de los artículos publicados, entre 1995 y 2004, en 94 revistas pertenecientes a las áreas de EF, Kinesiología, Educación y Ciencias Sociales, y Educación de la Salud y Médica, determinando que las revistas que cuentan con un mayor porcentaje de artículos publicados son revistas de EF como Journal of Teaching in Physical Education(14.38\%), Physical Educator (9\%), British Journal of Teaching Physical Education (7.63\%), European Physical Education Review (5.16\%), International Journal of Physical Education (4.72\%) y Journal of International Council for Health, Physical Education, Recreation, Sport and Dance (4.12\%). De otras disciplinas destacan Quest (6,75\%) y Research Quarterly for Exercise and Sport (4.1\%), ambas de Kinesiología.

El trabajo de Kirk (2010) describe las actuales tendencias de investigación de los autores europeos, revisando los trabajos publicados en la década 2000-2009 en cuatro revistas: European Physical Education
Review(EPER), Physical Education and Sport Pedagogy(PESP), Sport, Education and Society (SE\&S) y Journal of Teaching in Physical Education (JTPE). De un total de 665 artículos, 204 (un 30.67\%) son de autoría europea. EPER tiene el porcentaje más alto de trabajos de autores europeos con un 83\%, PESP un 62\% y SE\&S un 50\%. La norteamericana JTPE presenta el porcentaje más bajo con un 15\%. De los 10 países más importantes, el inglés es la lengua materna en tres de ellos (Inglaterra, Escocia e Irlanda). España ocupa el séptimo lugar con 11 trabajos en total, estando representada en cada una de las publicaciones.

En el panorama español, las aportaciones realizadas sobre PEF se han dirigido más a comunicar lo que está sucediendo en el panorama internacional y en ofrecer orientaciones a los investigadores españoles, que a presentar el estado de la investigación en el país (Barbero, 1994; Del Villar, 1996; Devís, 1993 y 2012; Fernández-Balboa, 1997). En este sentido, Rivera, Trigueros \& Pavesio (2003) señalan que, aunque se aprecia un incremento de la producción científica relacionada con la EF escolar, ésta sigue siendo escasa.

Ante la carencia de estudios específicos que contribuyan a situar el estado de la cuestión en España, el objetivo del artículo es realizar un análisis de las revistas con mayor producción en el ámbito de la PEF en la última década (2005-2014). Los objetivos concretos que se plantean son: a) determinar el porcentaje de artículos publicados sobre EF escolar en relación con la producción total de artículos publicados en cada una de las revistas analizadas y en la década estudiada; b) categorizar la producción encontrada según subáreas; y c) clasificar la producción según el tipo de artículo.

\section{Método}

\section{Unidad de análisis y materiales}

El análisis se centra en las revistas españolas más productivas en PEF en el campo de las CCAFD. Para su selección se partió de las revistas indizadas en WoS, Scopus o con el sello de calidad de la FECYT indicadas en la tabla 1. Por no orientarse hacia el ámbito pedagógico de este conjunto se eliminaron: Apunts. Medicina de l'Esport, Archivos de Medicina del Deporte y Revista Andaluza de Medicina del Deporte. Por otra parte, y ante la carencia de revistas disciplinares de PEF este conjunto se amplió, por juicio experto, a revistas que publican artículos relacionados con este ámbito. Un criterio que debían cumplir estas revistas era el estar disponibles a texto completo on-line. De esta forma, se añadieron Tándem. Didáctica de la Educación Física, Ágora para la educación física y el deporte y Revista Española de Educación Física y Deportes. El conjunto de revistas quedó conformado por un total de 13. Para el análisis se seleccionaron las cuatro que mayor porcentaje de artículos, relacionados con la PEF, publicaron en el trienio de 2012 a 2014 (tabla 2).

Las cuatro revistas finalmente seleccionadas para el estudio fueron:

- Ágora para la Educación Física y el Deporte (ISSN-e: 19897200; http://www5.uva.es/agora), que comenzó a publicarse en 2001. Editada por el Foro para la EF, la Actividad Física Recreativa y el Deporte de Castilla y León/ Valor Educativo de la Motricidad. Su periodicidad fue irregular al comienzo, pero desde 2009 publica tres

Tabla 2.

\begin{tabular}{|c|c|c|c|}
\hline Revista & $\begin{array}{c}\text { Total artículos } \\
\text { publicados }\end{array}$ & $\begin{array}{c}\text { Artículos de } \\
\text { PEF }\end{array}$ & $\%$ \\
\hline Tándem. Didáctica de la EF & 87 & 67 & 77.01 \\
\hline Ágora para la EF y el deporte & 55 & 21 & 28.18 \\
\hline Retos & 148 & 33 & 22.30 \\
\hline Revista Española de EF y Deportes & 78 & 12 & 15.38 \\
\hline Apunts. EF y Deportes & 107 & 13 & 12.15 \\
\hline Cultura, Ciencia, Deporte & 64 & 5 & 7.81 \\
\hline Revista Internacional de Medicina y CAFD & 144 & 10 & 6.94 \\
\hline European Journal of Human Movement & 60 & 4 & 6.67 \\
\hline $\begin{array}{l}\text { Revista Iberoamericana de Psicología del } \\
\text { Ejercicio y del Deporte }\end{array}$ & 60 & 3 & 5 \\
\hline RICYDE & 70 & 3 & 4.29 \\
\hline Journal of Human Sport and Exercise & 114 & 4 & 3.51 \\
\hline Revista de Psicología del Deporte & 172 & 6 & 3.49 \\
\hline Cuadernos de Psicología del Deporte & 121 & 2 & 1.65 \\
\hline
\end{tabular}


números al año (cuatrimestral), con artículos en idioma castellano y en inglés.

- Retos. Nuevas Tendencias en Educación Física, Deporte y Recreación (ISSN-e: 1988-2041; http://www.retos.org/nuevo.html), publicó su primer número en 2002. Pertenece a la Federación Española de Asociaciones de Docentes de Educación Física (FEADEF). Tiene una periodicidad semestral. Los artículos se publican principalmente en español, aunque también se admiten en inglés, francés y portugués.

- Revista Española de Educación Física y Deportes (ISSN-e: 2387-161X; http://www.reefd.es/index.php/reefd), fundada en 1949, es la revista decana del área, aunque su publicación a lo largo de los años ha sido muy irregular. La edita el Consejo General de Colegios Oficiales de Licenciados en Educación Física y en Ciencias de la Actividad Física y del Deporte (COLEF y CAFD). Actualmente tiene una periodicidad trimestral y publica los artículos en castellano. No es una revista electrónica como tal, sino una revista en formato papel con una versión en PDF de sus contenidos.

- Tándem, Didáctica de la Educación Física (ISSN: 2014-4768; http://tandem.grao.com), sacó su primer número en al año 2000. Se trata de la única revista del área publicada por una editorial comercial (Graó) y también de la única que en estos momentos es de pago, pues el resto de revistas han pasado en los últimos años a editarse en abierto (open access). En la actualidad tiene una periodicidad cuatrimestral. Se trata de una revista más profesional que científica, rasgo que afecta tanto a la extensión y formato de los artículos que publica como a su contenido, que como la propia revista indica en sus instrucciones «se caracterizarán por un tono divulgativo».

El acceso a los materiales tuvo lugar a través de la página web de las revistas y se realizó durante el mes de enero de 2015.

\section{Diseñoy procedimiento}

La investigación desarrollada tiene un enfoque cuantitativo, de carácter exploratorio y descriptivo, que incluye técnicas de investigación documental. Como investigación documental, tuvo como corpus de análisis todos los artículos originales y revisiones publicados por las cuatro revistas objeto del estudio. Se procedió a la consulta directa de de todos los artículos, que en total fueron 929. Tras un primer análisis se eliminaron 529 resultados que no se adecuaban a los criterios establecidos para el trabajo. Finalmente, la muestra objeto de estudio estuvo constituida por 400 artículos referidos al estudio de la Educación Física escolar dentro del periodo de búsqueda determinado y que fueron archivados en una base de datos para su posterior tratamiento informacional.

Para establecer las categorías temáticas se tomó el modelo de Silverman \& Ennis (1996), que ha sido también utilizado en otros trabajos de análisis de estudios de PEF (Silverman \& Manson, 2003; Kulinna, et al., 2009). En este modelo (figura 1) se definen tres subáreas en el campo de la PEF: el currículum, la formación del profesorado y la enseñanza. Los estudios sobre el currículum centran sus objetivos en lo que se enseña en la EF escolar y los factores que influyen en la configuración de sus contenidos. Las investigaciones sobre la formación del profesorado prestan su atención a las distintas etapas de preparación del profesorado de EF, desde la formación inicial, previa la docencia, a la

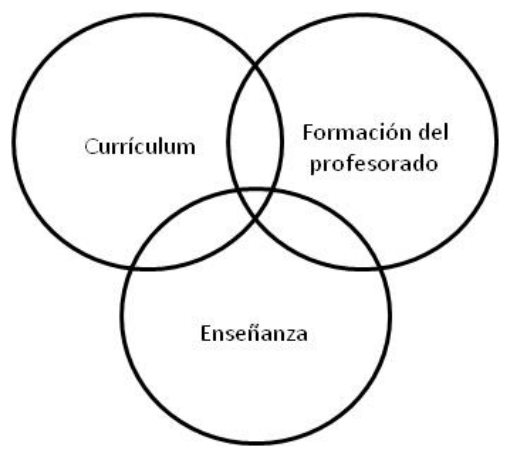

Figura 1. Subáreas de la PEF según Silverman \& Ennis (1996) formación permanente, una vez se está ejerciendo como docente. Por último, las investigaciones sobre la enseñanza focalizan sus objetivos en los procesos, las dinámicas sociales o los resultados de la EF. Como puede apreciarse en la figura 1, son tres subáreas que presentan intersecciones y espacios compartidos. Es decir, que, en algunas ocasiones, encontramos trabajos que pueden responder a más de una subárea.

El total de la muestra seleccionada fue analizada para determinar su pertenencia a alguna de las tres subáreas descritas. En segundo lugar, se procedió al análisis de contenido de cada uno de los artículos seleccionados y su clasificación dentro de alguno de los siguientes grupos: 1) estudios teóricos, históricos o ensayos; 2) revisiones bibliográficas; 3) descripción de experiencias educativas o de innovación; 4) investigaciones empíricas cuantitativas; 5) investigaciones empíricas cualitativas; 6) investigaciones empíricas mixtas.

Dos expertos (profesores de CCAFD) analizaron por separado cada uno de los artículos. Posteriormente, se compararon las clasificaciones obtenidas por ambos expertos. El porcentaje de acuerdo entre los codificadores fue de un $90 \%$. En aquellos casos en los que no coincidieron, se revisaron otra vez con la colaboración de un tercer experto, analizándose el contenido del artículo hasta que los tres expertos llegasen a una conclusión compartida.

La pretensión exclusivamente bibliométrica del estudio hizo que no se entrara a valorar la calidad de los trabajos. Para el almacenamiento de los datos y su análisis se utilizó el programa SPSS versión 22.0. Se utilizaron estadísticos descriptivos como frecuencias y porcentajes que se presentan en los resultados redondeados a dos decimales.

\section{Resultados}

\section{Artículos sobre Educación Física escolar}

En la tabla 3 se muestra el número total de artículos publicados por año en cada una de las revistas analizadas, así como el número y porcentaje de artículos respectivos sobre Educación Física escolar. De los 929 artículos publicados en la última década en las cuatro revistas analizadas, 400 son estudios sobre EF escolar. Es decir, el 43.06\% de la producción de estas revistas tiene que ver con la PEF, siendo la revista que más publica en este campo Tándem, con un 75.17\% del total de su producción. Seguida de Ágora (43.51\%), Retos (24.38\%) y Revista Española de Educación Física y Deportes (en adelante REEFD) (23.91\%).

Si nos fijamos en la evolución por años, se observan grandes oscilaciones en los porcentajes concretos de cada una de estas revistas. En Tándem destaca que el mayor porcentaje de artículos publicados fue en 2010, con un 90.32\%, mientras que el menor de esta revista fue en 2008, con un 58.06\%. En Ágora, cuando empezó en 2007 publicó un 87.5\% de artículos sobre EF escolar, mientras que en 2014 sólo publicó un 15.79\%. Retos publicó un 58.33\% en 2005, por un 11.36\% en 2010. Y REEFD publicó, un 76.92\% en 2008 y un 9.09\%, en 2014.

A lo largo de la década analizada, se observa una mengua general en la productividad en el campo de la PEF. Mientras los 4 primeros años se supera la mitad de artículos publicados, a partir de 2009 el porcentaje es menor advirtiéndose un decrecimiento en los últimos 4 años que llega al 28.36\% del último año analizado.

\section{Productividad por subáreas}

De los 400 artículos publicados en la última década sobre PEF en las cuatro revistas analizadas, casi la mitad focalizan su interés sobre la enseñanza (procesos, dinámicas sociales o resultados de la EF) y una cuarta parte en cuestiones relacionadas con el currículum (lo que se enseña en la EF escolar y los factores que influyen en la configuración de sus contenidos). Los artículos referidos a ambas posibilidades representan casi una décima parte de la producción. La formación (distintas etapas de preparación del profesorado EF, desde la formación inicial hasta la permanente) no llega a una décima parte. El resto de posibilidades obtienen porcentajes mucho más bajos (tabla 4).

Retos y Tándem se caracterizan por ser las dos revistas que más 
Tabla 3.

Número de artículos totales y de EF escolar, con su porcentaje respectivo, por año en cada una de las revistas analizadas

\begin{tabular}{|c|c|c|c|c|c|c|c|c|c|c|c|c|}
\hline & & 2005 & 2006 & 2007 & 2008 & 2009 & 2010 & 2011 & 2012 & 2013 & 2014 & Total \\
\hline \multirow[b]{2}{*}{ Tándem } & Artículos totales & 26 & 30 & 29 & 31 & 28 & 31 & 32 & 29 & 32 & 26 & 294 \\
\hline & $\begin{array}{l}\text { Artículos y \% de } \\
\text { PEF }\end{array}$ & $\begin{array}{c}18 \\
(69.23)\end{array}$ & $\begin{array}{c}24 \\
(80)\end{array}$ & $\begin{array}{c}23 \\
(79.31)\end{array}$ & $\begin{array}{c}18 \\
(58.06)\end{array}$ & $\begin{array}{c}18 \\
(64.29)\end{array}$ & $\begin{array}{c}28 \\
(90.32)\end{array}$ & $\begin{array}{c}25 \\
(78.13)\end{array}$ & $\begin{array}{c}24 \\
(82.76)\end{array}$ & $\begin{array}{c}24 \\
(75)\end{array}$ & $\begin{array}{c}19 \\
(73.08)\end{array}$ & $\begin{array}{c}221 \\
(75.17)\end{array}$ \\
\hline \multirow[b]{2}{*}{ Ágora } & Artículos totales & $*$ & * & 8 & 16 & 18 & 15 & 19 & 21 & 15 & 19 & 131 \\
\hline & $\begin{array}{l}\text { Artículos y \% de } \\
\text { PEF }\end{array}$ & * & * & $\begin{array}{c}7 \\
(87.5)\end{array}$ & $\begin{array}{c}7 \\
(43.75)\end{array}$ & $\begin{array}{c}9 \\
(50)\end{array}$ & $\begin{array}{c}4 \\
(26.67)\end{array}$ & $\begin{array}{c}9 \\
97.37)\end{array}$ & $\begin{array}{c}8 \\
(38.1)\end{array}$ & $\begin{array}{c}10 \\
(66.67)\end{array}$ & $\begin{array}{c}3 \\
(15.79)\end{array}$ & $\begin{array}{c}57 \\
(43.51)\end{array}$ \\
\hline \multirow[b]{2}{*}{ Retos } & Artículos totales & 12 & 8 & 21 & 28 & 37 & 44 & 22 & 31 & 50 & 67 & 320 \\
\hline & $\begin{array}{c}\text { Artículos y \% de } \\
\text { PEF }\end{array}$ & $\begin{array}{c}7 \\
(58.33) \\
\end{array}$ & $\begin{array}{c}4 \\
(50)\end{array}$ & $\begin{array}{c}4 \\
(19.05)\end{array}$ & $\begin{array}{c}9 \\
(32.14) \\
\end{array}$ & $\begin{array}{c}13 \\
(35.14)\end{array}$ & $\begin{array}{c}5 \\
(11.36) \\
\end{array}$ & $\begin{array}{c}3 \\
(13.64) \\
\end{array}$ & $\begin{array}{c}7 \\
(22.58) \\
\end{array}$ & $\begin{array}{c}12 \\
(24)\end{array}$ & $\begin{array}{c}14 \\
(20.9)\end{array}$ & $\begin{array}{c}78 \\
(24.38) \\
\end{array}$ \\
\hline \multirow{2}{*}{$\begin{array}{l}\text { Revista Española de } \\
\text { EF y Deportes }\end{array}$} & Artículos totales & 12 & 10 & 9 & 13 & 18 & 19 & 25 & 26 & 30 & 22 & 184 \\
\hline & $\begin{array}{l}\text { Artículos y \% de } \\
\text { PEF }\end{array}$ & $\begin{array}{c}3 \\
(25) \\
\end{array}$ & $\begin{array}{c}1 \\
(10)\end{array}$ & $\begin{array}{c}4 \\
(44.44) \\
\end{array}$ & 10 (76.92) & $\begin{array}{c}3 \\
(16.67) \\
\end{array}$ & $\begin{array}{c}4 \\
(21.05) \\
\end{array}$ & $\begin{array}{c}7 \\
(28) \\
\end{array}$ & $\begin{array}{c}7 \\
(26.92) \\
\end{array}$ & $\begin{array}{c}3 \\
(10) \\
\end{array}$ & $\begin{array}{c}2 \\
(9.09) \\
\end{array}$ & $\begin{array}{c}44 \\
(23.91) \\
\end{array}$ \\
\hline \multirow{2}{*}{$\begin{array}{l}\text { Totales entre las } 4 \\
\text { revistas }\end{array}$} & $\begin{array}{l}\text { Total artículos } \\
\text { publicados }\end{array}$ & 50 & 48 & 67 & 88 & 101 & 109 & 98 & 107 & 127 & 134 & 929 \\
\hline & $\begin{array}{c}\text { Total y \% de artículo } \\
\text { de PEF }\end{array}$ & $\begin{array}{c}28 \\
(56) \\
\end{array}$ & $\begin{array}{c}29 \\
(60.42) \\
\end{array}$ & $\begin{array}{c}38 \\
(56.72) \\
\end{array}$ & $\begin{array}{c}44 \\
(50) \\
\end{array}$ & $\begin{array}{c}43 \\
(42.57) \\
\end{array}$ & $\begin{array}{c}41 \\
(37.61) \\
\end{array}$ & $\begin{array}{c}44 \\
(44.90) \\
\end{array}$ & $\begin{array}{c}46 \\
(42.99) \\
\end{array}$ & $\begin{array}{c}49 \\
(38.58) \\
\end{array}$ & $\begin{array}{c}38 \\
(28.36) \\
\end{array}$ & $\begin{array}{c}400 \\
(43.06) \\
\end{array}$ \\
\hline
\end{tabular}

Tabla 4

\begin{tabular}{|c|c|c|c|c|c|}
\hline & Tándem & Ágora & Retos & REEFD & Totales \\
\hline Enseñanza & $\begin{array}{c}117 \\
(52.94)\end{array}$ & $\begin{array}{c}16 \\
(28.07) \\
\end{array}$ & $\begin{array}{c}47 \\
(60.26)\end{array}$ & $\begin{array}{c}11 \\
(25)\end{array}$ & $\begin{array}{c}191 \\
(47.75)\end{array}$ \\
\hline Currículum & $\begin{array}{c}49 \\
(22.17)\end{array}$ & $\begin{array}{c}21 \\
(36.84)\end{array}$ & $\begin{array}{c}19 \\
(24.36)\end{array}$ & $\begin{array}{l}22 \\
(50)\end{array}$ & $\begin{array}{c}111 \\
(27.75)\end{array}$ \\
\hline Formación & $\begin{array}{c}17 \\
(7.69)\end{array}$ & $\begin{array}{c}8 \\
(14.04) \\
\end{array}$ & $\begin{array}{c}6 \\
(7.69)\end{array}$ & $\begin{array}{c}4 \\
(9.09)\end{array}$ & $\begin{array}{c}35 \\
(8.75)\end{array}$ \\
\hline $\begin{array}{l}\text { Enseñanza y } \\
\text { currículum }\end{array}$ & $\begin{array}{c}28 \\
(12.67)\end{array}$ & $\begin{array}{c}4 \\
(7.02) \\
\end{array}$ & $\begin{array}{c}1 \\
(1.28) \\
\end{array}$ & $\begin{array}{c}5 \\
(11.36) \\
\end{array}$ & $\begin{array}{c}38 \\
(9.5)\end{array}$ \\
\hline $\begin{array}{l}\text { Formación y } \\
\text { currículum }\end{array}$ & $\begin{array}{c}6 \\
(2.71)\end{array}$ & $\begin{array}{c}3 \\
(5.26) \\
\end{array}$ & $\begin{array}{c}4 \\
(5.13)\end{array}$ & $\begin{array}{c}1 \\
(2.27) \\
\end{array}$ & $\begin{array}{c}14 \\
(3.5)\end{array}$ \\
\hline $\begin{array}{l}\text { Enseñanza y } \\
\text { formación }\end{array}$ & $\begin{array}{c}1 \\
(0.45) \\
\end{array}$ & $\begin{array}{c}5 \\
(8.77) \\
\end{array}$ & $\begin{array}{c}1 \\
(1.28)\end{array}$ & $\begin{array}{c}1 \\
(2.27) \\
\end{array}$ & $\begin{array}{c}8 \\
(2) \\
\end{array}$ \\
\hline $\begin{array}{l}\text { Enseñanza, } \\
\text { currículum y } \\
\text { formación }\end{array}$ & $\begin{array}{c}4 \\
(1.81)\end{array}$ & $\begin{array}{c}0 \\
(0)\end{array}$ & $\begin{array}{c}0 \\
(0)\end{array}$ & $\begin{array}{c}0 \\
(0)\end{array}$ & $\begin{array}{c}4 \\
(1)\end{array}$ \\
\hline Totales & 221 & 57 & 78 & 44 & 400 \\
\hline
\end{tabular}

artículos publican sobre enseñanza de la EF. Más de la mitad de sus artículos hacen referencia a esta subárea. Por su parte, REEFD y Ágora destacan en la subárea de currículum, llegando a la mitad de lo publicado la primera y a una tercera parte la segunda. En la subárea de formación los porcentajes son bajos y la única que supera una décima parte de lo publicado es Ágora.

\section{Tipo de artículos}

Como recoge la tabla 5, casi la mitad de los artículos sobre PEF publicados son estudios teóricos, históricos o ensayos. Les siguen los artículos sobre experiencias educativas o de innovación, que no superan la tercera parte de lo publicado. El conjunto de artículos relacionados con investigaciones empíricas se corresponde con una cuarta parte de lo publicado. Destaca también la enorme escasez de artículos de revisión.

Todas las revistas coinciden en que el mayor porcentaje de los artículos publicados en sus páginas son estudios teóricos, históricos o ensayos. Siendo la que más REEFD y la que menos Retos. Por su parte, la revista que más artículos publica sobre experiencias educativas o de innovación es Tándem. En cuanto a artículos de investigación cuantitativa la que más publica es Retos, mientras que de investigación cualitativa es Ágora. De los resultados presentados se desprende la siguiente caracterización de las revistas analizadas:

- Tándem se caracteriza por publicar estudios teóricos, históricos o ensayos, así como experiencias educativas o de innovación. Am-

Tabla 5

Tipo de artículo sobre PEF en cada una de las revistas analizadas.

\begin{tabular}{|c|c|c|c|c|c|}
\hline & Tándem & Ágora & Retos & REEFD & Totales \\
\hline $\begin{array}{l}\text { Estudios teóricos, } \\
\text { históricos o ensayos }\end{array}$ & $\begin{array}{c}100 \\
(45.25)\end{array}$ & $\begin{array}{c}30 \\
(52.63)\end{array}$ & $\begin{array}{c}28 \\
(35.9)\end{array}$ & $\begin{array}{c}27 \\
(61.36)\end{array}$ & $\begin{array}{c}185 \\
(46.25)\end{array}$ \\
\hline $\begin{array}{l}\text { Experiencias } \\
\text { educativas o de } \\
\text { innovación }\end{array}$ & $\begin{array}{c}94 \\
(42.53)\end{array}$ & $\begin{array}{c}7 \\
(12.28)\end{array}$ & $\begin{array}{c}6 \\
(7.69)\end{array}$ & $\begin{array}{c}5 \\
(11.36)\end{array}$ & $\begin{array}{l}112 \\
(28)\end{array}$ \\
\hline $\begin{array}{l}\text { Investigaciones } \\
\text { empíricas } \\
\text { cuantitativas }\end{array}$ & $\begin{array}{c}15 \\
(6.79)\end{array}$ & $\begin{array}{c}8 \\
(14.04)\end{array}$ & $\begin{array}{c}24 \\
(30.77)\end{array}$ & $\begin{array}{c}9 \\
(20.46)\end{array}$ & $\begin{array}{c}56 \\
(14)\end{array}$ \\
\hline $\begin{array}{l}\text { Investigaciones } \\
\text { empíricas cualitativas }\end{array}$ & $\begin{array}{c}8 \\
(3.62) \\
\end{array}$ & $\begin{array}{c}9 \\
(15.79) \\
\end{array}$ & $\begin{array}{c}8 \\
(10.26)\end{array}$ & $\begin{array}{c}1 \\
(2.27)\end{array}$ & $\begin{array}{c}26 \\
(6.5)\end{array}$ \\
\hline $\begin{array}{l}\text { Investigaciones } \\
\text { empíricas mixtas }\end{array}$ & $\begin{array}{c}4 \\
(1.81)\end{array}$ & $\begin{array}{c}2 \\
3.51)\end{array}$ & $\begin{array}{c}10 \\
(12.82)\end{array}$ & $\begin{array}{c}2 \\
(4.55)\end{array}$ & $\begin{array}{c}18 \\
(4.5)\end{array}$ \\
\hline $\begin{array}{l}\begin{array}{l}\text { Revisiones de } \\
\text { investigación }\end{array} \\
\end{array}$ & $\begin{array}{c}0 \\
(0) \\
\end{array}$ & $\begin{array}{c}1 \\
(1.75) \\
\end{array}$ & $\begin{array}{c}2 \\
(2.56) \\
\end{array}$ & $\begin{array}{c}0 \\
(0)\end{array}$ & $\begin{array}{c}3 \\
(0.75) \\
\end{array}$ \\
\hline Totales & 221 & 57 & 78 & 44 & 400 \\
\hline
\end{tabular}

bos tipos de artículos llegan a ocupar el 87.78\% de lo publicado en esta revista.

- EnÁgora la mitad de lo publicado se dedica a estudios teóricos, históricos o ensayos, mientras que la otra mitad prácticamente se reparte entre experiencias educativas o de innovación, investigaciones cuantitativas e investigaciones cualitativas.

- REEFD publica en su mayor parte, concretamente un 81.82\%, estudios teóricos, históricos o ensayos, e investigaciones de tipo cuantitativo.

Retos es la revista más orientada hacia la investigación empírica. Es la única en que este tipo de artículos supera la mitad de lo publicado, un $53.85 \%$ en concreto.

\section{Discusión y conclusiones}

En el contexto español, Devís, Valenciano, Villamón \& Pérez(2010), analizaron las disciplinas y temas de estudio de 16 revistas de las CCAFD durante el periodo de 1999-2005, situando la producción sobre didáctica en un $21.33 \%$ de lo publicado. Porcentaje sólo superado por el conjunto de especialidades médicas, con un $24.13 \%$. Nuestros resultados apuntan un cambio de tendencia. Si tenemos en cuenta que nuestro trabajo se centra en las cuatro revistas con mayor productividad en PEF y, sobre todo, si nos fijamos en la evolución de nuestros resultados, se observa una caída, en los últimos cuatro años, en el porcentaje de productividad en las revistas analizadas y donde sólo las revistas Tándem y Retos parecen mantener sus porcentajes. Resalta el bajo porcentaje (15.78\%) del último año analizado de la revista Ágora, pues nos referimos a una revista que, aunque tiene un carácter interdiciplinzar, en todos los años anteriores había mostrado un importante interés por lo pedagógico. La revista que más claramente muestra este cambio de tendencia es REEFD. En 2007 y 2008 mostraba un marcado interés por la producción pedagógica. En cambio, desde 2009 la brecha entre la producción total por año y la producción en cuanto a PEF, se hace cada vez mayor, hasta llegar a un porcentaje del 9.09\%. Esto podría indicar una nueva tendencia en esta revista que sería interesante comprobar en los próximos años.

En nuestro estudio hemos obtenido que casi la mitad de la productividad en PEF hace referencia a la enseñanza (47.75\%), seguida del currículum (27.75\%). La combinación de ambas subáreas es el tercer porcentaje mayor (9.5\%) y la formación y sus intersecciones con la enseñanza y el currículum, no llegan a una quinta parte de lo publicado (14.25\%). Hay mucha disparidad cuando comparamos estos resultados con otros estudios. Entre los que utilizan la misma categorización que nosotros encontramos el de Silverman \& Manson (2003), que analizaron 201 tesis doctorales sobre enseñanza de la EF (Teaching in Physical Education) durante el período de 1985 a 1999, descubriendo que el 36.67\% de las mismas se orientaron hacia la enseñanza, el 34.12\% hacia el currículum y el 29.19\% hacia la formación del profesorado. Kulinna et al. (2009), centraron su análisis sobre 1.819 trabajos de investigación sobre pedagogía de la EF, publicados entre 1995 y 2004, en 94 revistas diferentes de EF, Kinesiología, Educación y Ciencias Sociales y Educación de la Salud y Médica, constataron que el 65.31\% 
de los artículos atendieron a la enseñanza, el 19.24\% al currículum y el $15.45 \%$ a la formación del profesorado.

En lo referente a estudios que abordaron la misma temática que nosotros, pero utilizando distinta categorización, el trabajo de Ward \& Ko (2006) centra su análisis sobre una de las revistas referentes internacionalmente en el campo de la PEF, se trata de Journal of Teaching in Physical Education (JTPE). Analizaron las tendencias de publicación en la misma duranteel período de 1981 a 2005. Dentro de la categorización que utilizaron, constataron que una quinta parte de los artículos hacían referencia a la formación del profesorado, otra quinta parte a la pedagogía y poco menos de una décima parte al currículum. Más en nuestra línea, aunque también con otra categorización, destaca el análisis comparativo realizado por Kirk (2010) sobre 4 de las revistas de mayor repercusión en el ámbito de la pedagogía dentro de nuestra área de conocimiento: European Physical Education Review, Physical Education and Sport Pedagogy, Sport, Education and Society y JTPE, durante la década 2000 a 2009. La mitad de los trabajos (50.90\%) hicieron referencia al alumnado y su aprendizaje, un $39.22 \%$ al profesorado en cuanto a los procesos de enseñanza utilizados y a la formación del mismo, un 19.12\% sobre currículum o conocimiento y un 30.39\% combinaron varias de estas variables mencionadas.

En cuanto al tipo de artículos que hemos encontrado en nuestro trabajo, la mayor parte son estudios teóricos, históricos o ensayos (46.25\%) y experiencias educativas o de innovación (28\%). Los artículos de investigación empírica cubren una cuarta parte (25\%) de lo publicado y los artículos de revisión tienen una presencia testimonial (0.75\%). Retos es la es la única revista donde los artículos de investigación superan la mitad de lo publicado (53.9\%). En el panorama internacional la situación en el campo pedagógico es diferente, predominando los artículos de investigación empírica. Silverman \& Skonie(1997) realizaron una revisión de los trabajos sobre enseñanza de la EF (Teaching in Physical Education) publicados, entre 1980 y 1994, en revistas y actas de congresos indizadas en las bases de datos de Current Contents, ERIC o Sport Discus. Obtuvieron un total de 179 trabajos, la mayoría de los cuales responden a investigaciones de tipo cuantitativo y centradas en la eficacia docente. El anteriormente citado trabajo de Silverman \& Manson (2003), constataron que tres cuartas partes de las tesis doctorales analizadas eran investigaciones de corte cuantitativo. El también antes citado trabajo de Ward \& Ko (2006), señala que el 68\% de los artículos publicados en JTPE son de investigación, de los cuales el 40\% son cuantitativos, $18 \%$ cualitativos, $6 \%$ utilizan una metodología mixta y el 4.5\% son diseños de un sólo caso (single-subject designs). Posteriormente, Hemphill, Richards, Templin \& Blankenship, (2012) realizaron un análisis de contenido de la investigación cualitativa publicada entre 1998 y 2008 en JTPE, observando que los trabajos cualitativos o mixtos (que combinan la metodología cuantitativa y cualitativa) superan la mitad de los artículos publicados en esta revista. A este respecto, cabe destacar que en nuestro estudio hemos encontrado que la revista Ágora publica un 19.3\% de artículos relacionados con la investigación cualitativa y Retos un $23.1 \%$. Aunque son cifras muy alejadas del porcentaje de lo que está publicando JTPE, también lo están de la situación de la investigación cualitativa en las revistas españolas de CCAFD. Molina, Villamón \& Úbeda (2015), a partir del análisis de 1057 artículos publicados entre 2007 y 2011 en revistas españolas de CCAFD indizadas en Scopus, encuentran que sólo un 3.5\% son de investigación cualitativa.

Los resultados que hemos obtenido, según subárea y tipo de artículos, también han servido para presentar una caracterización del perfil de las cuatro revistas que puede servir de orientación a los investigadores, del ámbito de la PEF, para determinar el envío de sus artículos para publicación. Retos parece estar reforzando un perfil más científico, más aún cuando acaba de ser indizada en 2016 en Emerging Sources Citation Index de WoS y en Scopus y, a juzgar por los resultados, creemos que puede ser la revista referente española en el campo de la PEF.

Señalar por último que, si se quiere que el campo de la PEF en España tenga una situación académico-científica similar a la de los países anglófonos, es fundamental conseguir que se publique un mayor porcentaje de artículos originales de investigación empírica (cuantitativa, cualitativa o mixta) en detrimento de los estudios teóricos, históricos o ensayos. Esta labor atañe a los equipos editoriales de las revistas, pero también a los investigadores del ámbito estudiado.

\section{Referencias}

Barbero, J.I.(Ed.) (1994). Investigación alternativa en educación física. Málaga: Unisport Andalucía.

Del Villar, F. (Coord.) (1996). La investigación en la enseñanza de la educación física. Cáceres: Universidad de Extremadura.

Devís, J. (1994). Introducción crítica a la investigación positivista en la enseñanza de la educación física. En J.I. Barbero (Coord.): Investigación alternativa en educación física (pp.31-72). Málaga: Unisport.

Devís, J. (2012). La investigación sociocrítica en la educación física. Estudios Pedagógicos, 38(1), 125-153.

Devís, J., Valenciano, J., Villamón, M. \& Pérez-Samaniego, V. (2010). Disciplinas y temas de estudio en las ciencias de la actividad física y el deporte. Revista Internacional de Medicina y Ciencias de la Actividad Física y el Deporte, 10(37), 150-166.

Fernández-Balboa, J.M.(1997). La investigación en la educación física española: un índice para el futuro. Apunts: Educación física y deportes, 50, 100-106.

Hemphill, M., Richards, K., Templin, T. \& Blankenship, B. (2012). A ContentAnalysis of Qualitative Research in the Journal of Teaching in Physical Education from 1998 to 2008. Journal of Teaching in Physical Education, 31(3), 279-287.

Kirk, D. (2010). Why research matters: Current status and future trends in physical education pedagogy. Movimento, 16(2), 11-43.

Kulinna, P., Scrabis-Fletcher, K., Kodish, S., Phillips, S. \& Silverman, S. (2009). A Decade of Research Literature in Physical Education Pedagogy. Journal of Teaching in Physical Education, 28(2), 119140.

MEC (1991). Real Decreto 1440/1991, de 30 de agosto, por el que se establece el titulo universitario oficial de Maestro, en sus diversas especialidades y las directrices generales propias de los planes de estudios conducentes a su obtención. BOE, 244, de 11 de octubre, 33003-33018.

MEC (1992). Real Decreto 1423/1992, de 27 de noviembre, sobre incorporación a la universidad de las enseñanzas de Educación Física. BOE, 307, de 23 de diciembre, 43641-43643.

Molina, P., Villamón, M. \& Úbeda, J. (2015). La investigación cualitativa en las revistas españolas de ciencias del deporte (2007-2011). Revista de Psicología del Deporte, 24(1), 29-36.

Rivera, E., Trigueros, C. \& Pavesio, M. (2003). Líneas de investigación en Educación Física escolar. Ágora para la Educación Física y el Deporte, 2-3, 75-8.

Silverman, S. \& Ennis, C. (1996). Enhancing learning: An introduction. In S. Silverman \& C. Ennis (Eds.), Student learning in physical education: Applying research to enhance instruction (pp. 3-8). Champaign, IL: Human Kinetics.

Silverman, S. \& Manson, M. (2003). Research on Teaching in Physical Education Doctoral Dissertations: ADetailed Investigation of Focus, Method, and Analysis. Journal of Teaching in Physical Education, 22(3), 280-297.

Silverman, S. \& Skonie, R. (1997). Research on Teaching in Physical Education: AnAnalysis of Published Research. Journal of Teaching in Physical Education, 16(3), 300-311.

Valenciano, J., Devís, J. \& Beltrán, V. (2008). La investigación y las revistas científicas de la actividad física y el deporte en España. Revista Fuentes, 8, 209-218.

Villamón, M., Job, I., Valenciano, J. \& Devís, J. (2012). Estudio comparativo de cinco revistas de Ciencias del Deporte indizadas en WoS. Revista de Psicología del Deporte, 21(2), 281-287.

Ward, P. \& Ko, B. (2006). Publication Trends in the Journal of Teaching in Physical Education from 1981 to 2005. Journal of Teaching in Physical Education, 25(3), 266-280. 